\title{
Exploring ultra-fast charge transfer and vibronic coupling with N 1s RIXS maps of an aromatic molecule coupled to a semiconductor
}

\author{
James N. O'Shea, 1 (a) Karsten Handrup, ${ }^{2}$ Robert H. Temperton, ${ }^{1}$ Andrew J. Gibson, ${ }^{1}$ \\ Alessandro Nicolaou, ${ }^{3}$ and Nicolas Jaouen ${ }^{3}$ \\ 1) School of Physics, University of Nottingham, NG7 2RD, \\ $U K$ \\ 2) Synchrotron Radiation Research, Department of Physics, Lund University, \\ Box 118, SE-221 00 Lund, Sweden \\ 3) Synchrotron SOLEIL, Saint-Aubin, BP 48, 91192 Gif-sur-Yvette, \\ France
}

We present for the first time two-dimensional resonant inelastic x-ray scattering (RIXS) maps of multilayer and monolayer biisonicotinic acid adsorbed on the rutile $\mathrm{TiO}_{2}(110)$ single crystal surface. This enables the elastic channel to be followed over the lowest unoccupied molecular orbitals resonantly excited at the $\mathrm{N} 1 s$ absorption edge. The data also reveals ultra-fast intramolecular vibronic coupling, particularly during excitation into the LUMO-derived resonance. Both elastic scattering and the vibronic coupling loss features are expected to contain the channel in which the originally excited electron is directly involved in the core-hole decay process. This allows RIXS data for a molecule coupled to a wide bandgap semiconductor to be considered in the same way as the core-hole clock implementation of resonant photoemission spectroscopy (RPES). However, contrary to RPES measurements, we find no evidence for depletion of the participator channel under the conditions of ultrafast charge transfer from the molecule to the substrate densities of states, on the timescale of the core-hole lifetime. These results suggest that the radiative core-hole decay processes in RIXS are not significantly modified by charge transfer on the femtosecond timescale in this system.

a)Electronic mail: J.Oshea@nottingham.ac.uk 


\section{INTRODUCTION}

Ultra-fast electron transfer between a molecule and a surface to which it is coupled plays a key role in light-harvesting devices such as dye-sensitised solar cells ${ }^{11}$ and water-splitting photoelectrochemical cells $s^{2}$ - to name just a couple of examples. A particularly elegant way to probe these charge transfer processes that can often occur on the low femtosecond timescale, is the use of resonant core-level spectroscopy - the most widely applied implementation being resonant photoemission (RPES).

In a solar cell, a light-harvesting molecule absorbs a photon of visible light through the excitation of an electron from the highest-occupied molecular orbital (HOMO) to the lowest unoccupied molecular orbital (LUMO). In RPES, the LUMO (and higher energy molecular orbitals) is instead populated by the resonant excitation of a core-electron through the absorption of a soft x-ray photon tuned to the specific energy of that resonance. This excitation is atom-specific because the transition probability is proportional to the overlap of the unoccupied molecular orbital with the specific core-level being probed. Moreover, the core-hole has an intrinsically short lifetime $\frac{3}{3}$ opening up the possibility to probe what

happens to the excited electron on the low femtosecond timescale. ${ }^{4}$ If the electron transfers away from the molecular orbital on the timescale of the core-hole lifetime then the core-hole decay channel in which this electron is a direct participant will be depleted.

In RPES this participator (participant) ${ }^{5}$ channel is an Auger-like transition in which the originally excited electron fills the core-hole and the energy is released non-radiatively by the emission of an electron from one of the highest occupied molecular orbitals, leaving the molecule in a one-hole final state identical to direct photoemission of that occupied orbital. This core-hole clock implementation of resonant photoemission has been reviewed in detail by Brühwiler et $a l^{[4}$ and led to the experimental observation of sub-3 fs charge transfer from the aromatic molecule biisonicotinic acid to the conduction band of a single crystal rutile $\mathrm{TiO}_{2}(110)$ substrate. ${ }^{6}$ This is an ideal model for a dye-sensitised light harvesting surface since it functions as the anchor ligand of many organometallic dye molecules. ${ }^{1}$ It is has been shown to bond to the $\mathrm{TiO}_{2}(110)$ surface in a $2 \mathrm{M}$-bidentate fashion through the deprotonation of the carboxyl groups $\mathrm{s}^{7}$ as shown in Fig. 1. The strong chemical coupling between the molecule and the surface provides an efficient pathway for charge injection into the oxide conduction band. RPES has since been extended beyond the ligand molecules of 


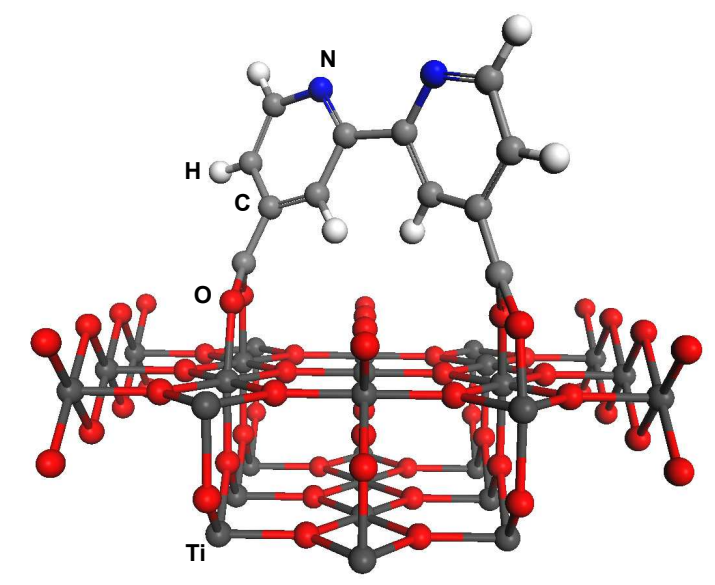

FIG. 1. Geometry of the biisonicotinic acid molecule chemisorbed in a 2M-bidentate fashion through the deprotonation of both carboxylic groups. The titanium and oxygen atoms in the rutile $\mathrm{TiO}_{2}(110)$ surface are represented by red and dark gray spheres, respectively. The nitrogen, oxygen, carbon and hydrogen atoms of the molecule are represented by blue, red, light gray, and white spheres, respectively.

organometallic complexes to the dyes themselves to probe the charge transfer dynamics on the femtosecond timescale. ${ }^{8}[10$

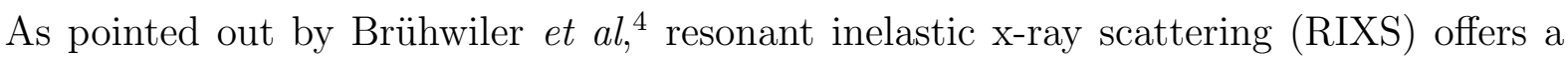
similar potential for observing the charge transfer dynamics of adsorbed molecules as RPES. A schematic illustration of the electronic transitions involved is shown in Fig. 2. Following the resonant excitation of a core-electron to an unoccupied valence state through $\mathrm{x}$-ray absorption (Fig. 2a), the core-hole can decay via two channels. In the participator channel (Fig. 2b) the core-hole is filled by the originally excited electron from the unoccupied valence state, resulting in the emission of an x-ray photon of the same energy as the x-ray absorption. Participator decay therefore results in elastic scattering. Alternatively, in the the spectator channel (Fig. 22) the core-hole is filled by an electron in one of the occupied valence states, resulting in the emission of an x-ray photon of lower energy than the one absorbed. Spectator decay therefore results in inelastic scattering.

If we now couple the molecule to a surface such that ultra-fast charge transfer can occur to the conduction band of the substrate on the timescale of the core-hole lifetime, both the participator and spectator channels will be depleted in favour of 'normal' x-ray emission (Fig. 2d). If the participator channel can be clearly differentiated in RIXS then it can be 


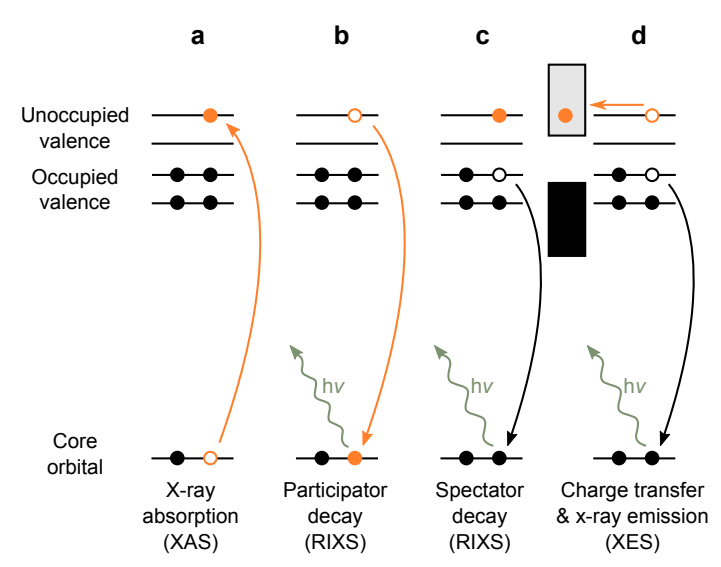

FIG. 2. Relevant processes. a XAS resonant excitation of a core electron to an unoccupied state (molecular orbital). b Participator decay in which the core-hole is filled by the originally excited electron resulting in elastic scattering and $\mathbf{c}$ spectator decay in which the core-hole is filled by a different valence electron resulting in inelastic scattering. $\mathbf{d}$ If the molecular orbital couples to the substrate empty states (conduction band) charge transfer of the electron can take place, which competes with RIXS, resulting in XES.

used in the same way as in RPES to provide information on the degree of localisation of the core electron excited to different unoccupied molecular orbitals - on the timescale of the core-hole lifetime.11

In this paper we present for the first time two-dimensional $\mathrm{N} 1 s$ RIXS maps of multilayer and monolayer biisonicotinic acid adsorbed on the rutile $\mathrm{TiO}_{2}(110)$ single crystal surface, allowing the participator channel to be followed over the lowest unoccupied molecular orbitals. The data also reveals ultra-fast vibronic coupling on the timescale of the core-hole lifetime, leading to an strong inelastic component of the participator channel.

\section{METHOD}

RIXS measurements were performed at the AERHA spectrometer ${ }^{12}$ at the SEXTANTS beamline at Synchrotron SOLEIL.13 Multilayer samples were prepared in a separate UHV system in our home laboratory equipped with a Scienta R3000 analyser and dual anode (Al $\mathrm{k} \alpha, \mathrm{Mg} \mathrm{k} \alpha$ ) x-ray source. Substrates were rutile $\mathrm{TiO}_{2}(110)$ single crystals (Pi-Kem, UK) cleaned by repeated cycles of sputtering at $2 \mathrm{kV} \mathrm{Ar}^{+}$followed by $1 \mathrm{kV} \mathrm{Ar}^{+}$, and annealing in UHV to $875 \mathrm{~K}$ until no C $1 s$ signal was detected in the XPS. Multilayers of bi-isonicotinic acid 
(4,4-dicarboxy-2,2-bipyridine from Alfa Aesar), were deposited onto the prepared substrates held at room temperature by sublimation from a Knudsen-type cell evaporation source until no Ti $2 p$ signal was detected in the XPS. This procedure produces atomically flat surfaces suitable for RIXS measurements with low/negligible contribution of the diffuse scattering to the elastic line intenisty. The multilayer samples were then transferred into the analysis chamber of the AERHA spectrometer where the base pressure was $1 \times 10^{-9}$ mbar. For RIXS measurements on the multilayers, samples were used as prepared. For RIXS measurements of the monolayers, these were prepared by annealing fresh multilayer samples in-situ to $500 \mathrm{~K}$ to desorb the physisorbed multilayer to leave just the chemisorbed monolayer (see Fig. 11 ${ }^{7}$ The thickness of the multilayer films is estimated from the XPS to be around $10 \mathrm{~nm}$. Due to the increased penetration depth of x-rays compared to photoelectrons, there will be a small contribution of the first monolayer in the multilayer RIXS measurements. However, this is estimated to be at most a few percent of the overall signal and there are no features in the multilayer RIXS data presented here that can be clearly attributed to this contribution.

The incident beam at SEXTANTS is focused to a spot size of $2(\mathrm{v}) \times 100(\mathrm{~h}) \mu \mathrm{m}$. To prevent beam damage of the molecules, the sample was continuously moved during the measurements at a rate that was shown to not give rise to changes between successive fast $\mathrm{x}$-ray absorption spectroscopy (XAS) scans. Due to the very low x-ray emission signal monolayer $\mathrm{N}$ 1s RIXS maps were accumulated over more than one sample and the total x-ray emission intensity detected at each absorption energy normalized to the corresponding fluorescenceyield XAS intensity measured using an MCP detector. The photon energy scale of the RIXS spectrometer was calibrated by measuring the elastic peak at $5 \mathrm{eV}$ increments across the whole detector range using linear vertical polarization of the incoming beam to maximise the elastic (Rayleigh) scattering intensity. For the RIXS maps and XAS over the N $1 s$ absorption edge the beam was linearly polarized in the horizontal plane and impinging on the sample with an incidence angle of $35^{\circ}$ with respect to the surface. The spatial distribution of the three lowest unoccupied molecular orbitals in the core-excited state for both the isolated and adsorbed biisonicotinic acid molecules have been calculated by DFT and published previously ${ }^{14}$ These $\pi^{*}$ orbitals will have a significant absorption cross-section when the polarisation of the incoming beam cuts through the plane of the molecule, which is the case for the ordered monolayer (Fig. 1) at the chosen experimental geometry in addition to the disordered multilayer. The overall measurement resolution was set to $200 \mathrm{meV}$ (com- 


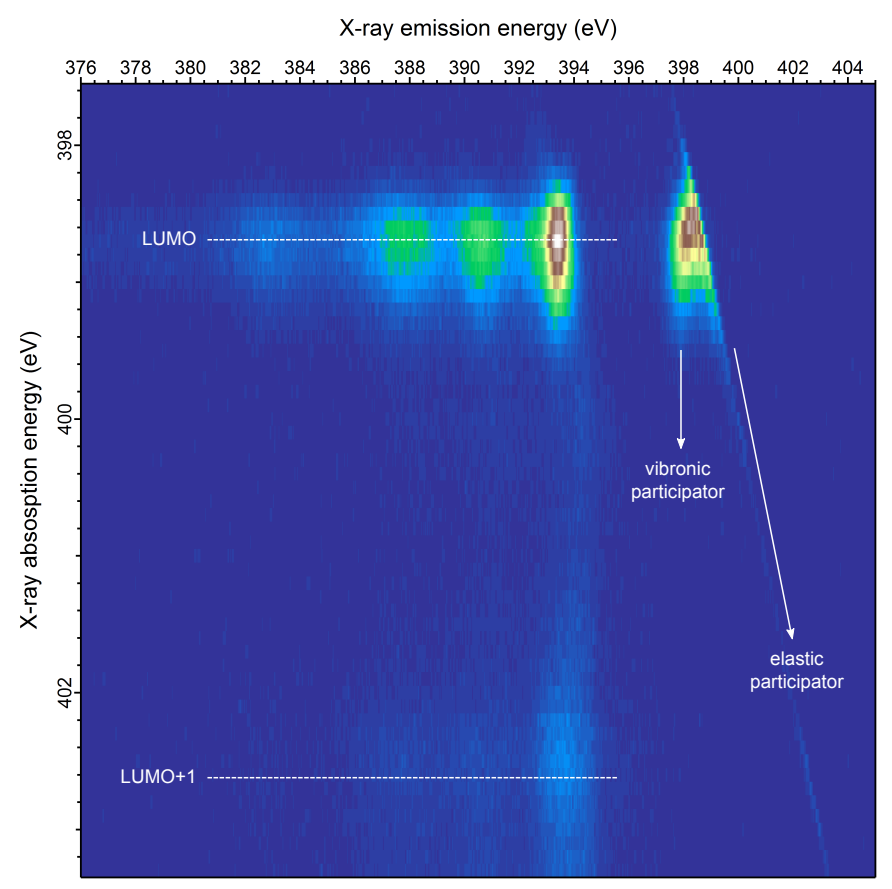

FIG. 3. N $1 s$ RIXS map for a biisonicotinic acid multilayer (deposited to a thickness where photomemission from the underlying $\mathrm{TiO}_{2}(110)$ surface is not visible in XPS). The features observed below $396 \mathrm{eV}$ emission energy are attributed to spectator decay (inelastic scattering) and those above $397 \mathrm{eV}$ emission energy to participator decay (elastic scattering and small inelastic losses due to vibronic coupling). These features are observed at the LUMO and LUMO+1 resonances at $398.7 \mathrm{eV}$ and $402.6 \mathrm{eV}$, respectively. Total acquisition time for the map was approximately 9 hours.

pared to the optimum resolution of $110 \mathrm{meV}$ ) as a compromise between energy resolution and spectrometer transmission to keep the molecule below the radiation damage threshold. For the multilayer RIXS map, each line is an x-ray emission spectrum accumulated for 10 mins at $0.1 \mathrm{eV}$ intervals of absorption energy. For the monolayer RIXS map each line is the average of between 3 and 12 spectra, each accumulated for $10 \mathrm{mins}$, at $0.2 \mathrm{eV}$ intervals.

\section{RESULTS AND DISCUSSION}

The RIXS map measured over the N $1 s$ absorption edge of multilayer biisonicotinic acid is shown in Fig. 3. The horizontal axis of the map represents the x-ray emission energy, while the vertical axis represents the x-ray excitation energy (absorption energy). Two main bands of emission features are observed at absorption energies of $398.7 \mathrm{eV}$ and $402.6 \mathrm{eV}$. 


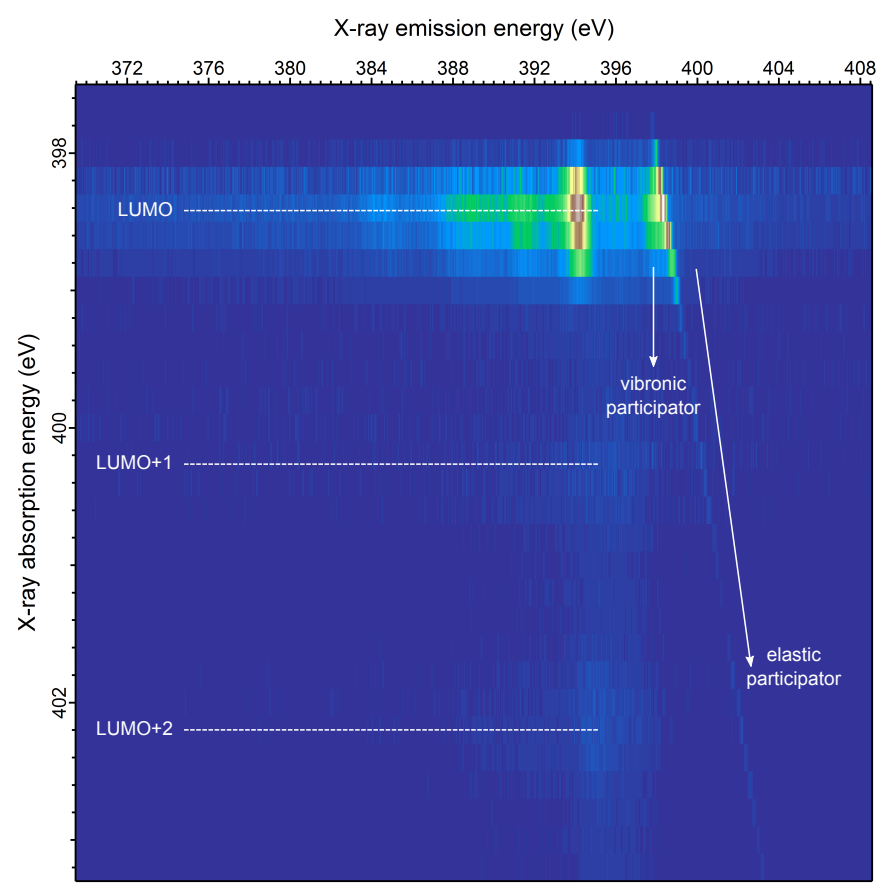

FIG. 4. N $1 s$ RIXS map for a biisonicotinic acid monolayer adsorbed on the rutile $\mathrm{TiO}_{2}(110)$ single crystal surface. The features observed below $396 \mathrm{eV}$ emission energy are attributed to inelastic scattering of the x-rays and those above $397 \mathrm{eV}$ emission energy to elastic scattering and small inelastic losses due to vibronic coupling. These features are observed at the LUMO, LUMO+1 and LUMO+2 resonances at $398.4 \mathrm{eV}, 400.2 \mathrm{eV}$ and $402.3 \mathrm{eV}$, respectively. Total acquisition time for the map was approximately 24 hours.

These correspond to the LUMO and LUMO+1 resonances of the molecule, respectively. The features within these bands below around $396 \mathrm{eV}$ emission energy are attributed to inelastic x-ray scattering arising from the $\mathrm{N} 1 s$ core-hole being filled by valence electrons from the occupied molecular orbitals. This is the projection of the occupied densities of states of the molecule onto the nitrogen core-level within the context of the dipole selection rule. These features are therefore a measure of the occupied $\pi$ orbitals derived from the nitrogen valence electrons. Since the molecules are decoupled from the underlying oxide surface in thick physisorbed multilayer, the core-excited electrons will be localised in the unoccupied molecular orbital many orders longer than the timescale of the core-hole lifetime as there are no empty states available to tunnel into. The inelastic scattering features below $396 \mathrm{eV}$ are therefore attributed to the spectator decay process illustrated in Fig. 26.

The narrow diagonal feature observed on the right hand side of Fig. 3 is a feature of 
equivalent absorption and emission energy and therefore attributed to elastic scattering. Diffuse reflection of the incoming beam will inevitably contribute to some extent to the elastic peak, however the experiment has been configured to minimise this by avoiding the specular reflection angle and using horizontally polarised light. Moreover, this contribution will be constant over the absorption energy range probed so that changes in the intensity of the elastic peak can be attributed to changes in the participator decay process illustrated in Fig. 2 b.

A decrease in the intensity of the participator channel can arise for two reasons that are essential to disentangle. The first is charge transfer of the participator electron out of the molecular orbital on the timescale of the core-hole lifetime. The second is a decrease in the probability for participator decay relative to spectator decay for the higher-energy molecular orbitals. Since ultra-fast charge transfer is not possible for molecules decoupled from the substrate densities of states, we can assess the orbital-specific participator probability in the absence of charge transfer from the multilayer RIXS.

Such a quantification requires identification of all the x-ray photons emitted from a participator decay process, including those that are not strictly elastic. This is relevant in the context of the multilayer RIXS data shown in Fig. 3 as considerable intensity is observed to the low energy side of the elastic line observed over the LUMO resonance. This feature has an intensity centred around an emission energy of $398 \mathrm{eV}$. There are however, no occupied molecular orbitals at this energy since this corresponds to the onset of the LUMO resonance. This feature is instead attributed to intra-molecular vibronic coupling and interpreted in a Franck-Condon scheme where the excited electron rapidly couples to the lowest vibrational level of the LUMO-derived resonance. The electron then decays to fill the core-hole, with the emission of a photon energy corresponding to the difference between this lowest vibrational state and the $\mathrm{N} 1 s$ ground state.

It follows that this intra-molecular vibronic coupling must occur on on the same order or faster than the timescale of the core-hole lifetime of a few fs. Ultra-fast vibronic dynamics have previously been observed in the $\mathrm{C} 1 s \mathrm{RPES}^{15[16}$ and RIXS ${ }^{15}$ of $\mathrm{C}_{60}$ multilayers and also in $\mathrm{N} 1 s$ RIXS maps of aqueous $\mathrm{NH}_{3} \cdot{ }^{17}$ It also follows that since the emission associated with the vibronic coupling arises from a transition involving the originally excited electron, that these photons must be considered part of the participator decay channel.

The RIXS map measured over the $\mathrm{N} 1 s$ absorption edge of monolayer biisonicotinic acid 
on the rutile $\mathrm{TiO}_{2}(110)$ single crystal surface is shown in Fig. 4. Here, three main bands of emission features are observed at absorption energies of $398.4 \mathrm{eV}, 400.2 \mathrm{eV}$ and $402.3 \mathrm{eV}$. These correspond to the LUMO, LUMO+1 and LUMO+2 resonances of the molecule, respectively. The LUMO and LUMO+2 of the chemisorbed molecules correspond to the LUMO and LUMO+1 observed for the physisorbed multilayer. The LUMO+1 observed at $400.2 \mathrm{eV}$ for the monolayer is derived from the adsorption bond and has previously been observed both experimentally and computationally for a biisonicotinic acid monolayer on the rutile $\mathrm{TiO}_{2}(110)$ surface. ${ }^{7}$ The interpretation of the monolayer RIXS is largely the same as for the multilayer, with features below $396 \mathrm{eV}$ emission energy attributed to inelastic scattering, and those above to the elastic scattering. The broad intensity around the elastic line at the LUMO resonance is again attributed to vibronic coupling within the molecule in the core-excited state.

The key difference between the monolayer and multilayer is that in the monolayer some molecular orbitals can couple to empty densities of states in the oxide substrate, opening up the possibility of molecule-surface charge transfer. From combined XAS and photoemission

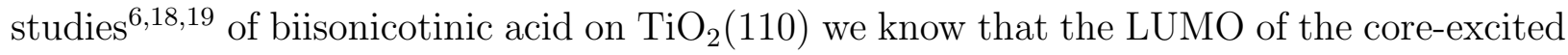
molecule lies below the conduction band edge of the oxide and therefore overlaps with the bandgap. For the LUMO state then we still expect to observe an intense participator channel since an electron excited to this state cannot tunnel away into empty states in the surface. The vibronic coupling feature at the LUMO resonance is consistent with this localisation on the timescale of the core-hole lifetime. The LUMO+1 and LUMO+2 states however, lie well above the conduction band edge of the $\mathrm{TiO}_{2}(110)$ surface and have been shown through RPES to undergo ultra-fast charge transfer into the surface on the low femtosecond timescale. ${ }^{6[18}$

Integrating over the participator region of the RIXS for both the multilayer and monolayer as a function of absorption energy, we compare the relative intensities of the participator channel for each unoccupied molecular orbital compared to the x-ray absorption (XAS). Since charge transfer out of the LUMO is not allowed for either the multilayer (as the molecules are decoupled from the substrate densities of states) or the monolayer (as it lies energetically below the conduction band) the participator RIXS of both surfaces can be normalised to the intensity of the LUMO in the XAS. These spectra are shown in Fig. 5.

Rearranging the equations given by Brühwiler et al, $\frac{4}{4}$ enables us to estimate a charge 

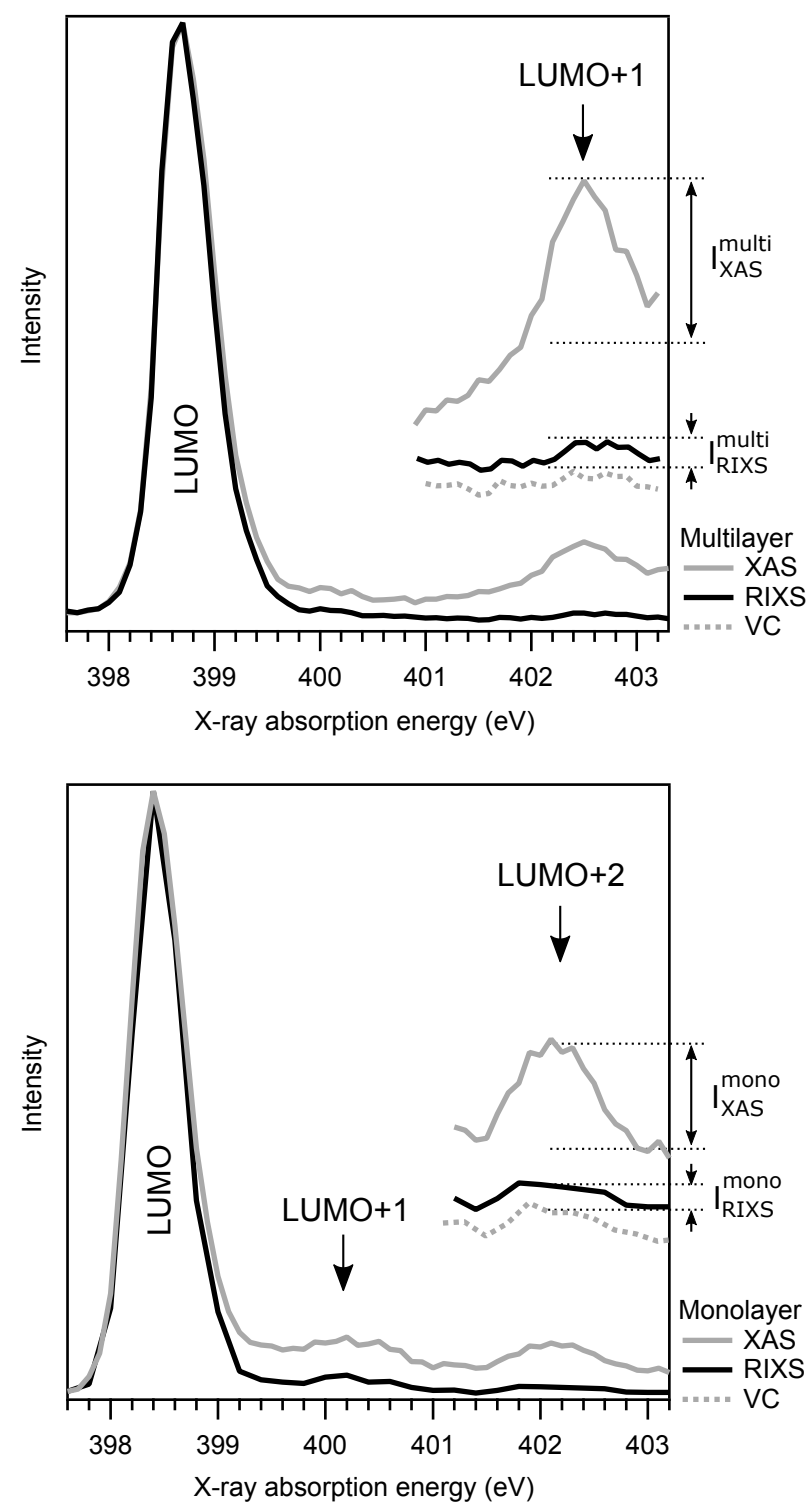

FIG. 5. Integrations over the participator region of the $\mathrm{N} 1 s$ RIXS for multilayer (a) and monolayer (b) biisonicotinic acid on the $\mathrm{TiO}_{2}(110)$ surface, compared to XAS. The integration window for the RIXS at each absorption energy was $2 \mathrm{eV}$ wide starting from the high emission energy side of the elastic line, and $1.7 \mathrm{eV}$ wide for the vibronic coupling feature (VC) starting from the low emission energy side of the elastic line. All spectra have been normalised to the height of the LUMO which lies energetically below the conduction band and therefore cannot participate in charge transfer into states in the underlying oxide surface.

transfer time $\tau_{C T}$ for the LUMO+2 state of the coupled molecule from the core-hole lifetime $\tau_{C}$, 


$$
\tau_{C T}=\tau_{C} \frac{\frac{I_{R I X S}^{m o n o}}{I_{X A S}^{m o n o}}}{\frac{I_{R I X S}^{m u l t i}}{I_{X A S}^{m u l t i}}-\frac{I_{R I X S}^{m o n o}}{I_{X A S}^{m o n o}}}
$$

where $\frac{I_{R I X S}^{m u l t i}}{I_{X A S}^{m u l t i}}$ is the ratio of participator signal in the RIXS to the total x-ray absorption measured in the fluorescence yield XAS for the multilayer, and $\frac{I_{R I X S}^{\text {mono }}}{I_{X A S}^{\text {mono }}}$ is the corresponding ratio for the monolayer. Replacing the intensity ratios in Eqn. 1 with the values extracted from the integrations in Fig. 5, and setting to $\tau_{C}=6 \mathrm{fs}, \frac{3}{3}$ the charge transfer timescale for $\mathrm{LUMO}+2$ could, in principle, be estimated. However, the ratios extracted from an integration of the RIXS over a $2 \mathrm{eV}$ range covering both the elastic peak and the vibronic coupling are essentially the same within experimental uncertainty (0.2 \pm 0.1$)$. For completeness, Fig. 5 also shows the integration performed over just the vibronic coupling region on its own (not including the elastic line) with no change in the result. This corresponds to a localisation of the electron in the LUMO+2 on a timescale orders of magnitude longer that the corehole lifetime, in direct contradiction to previous experimental evidence for ultra-fast charge transfer based on the RPES participator channel.

Charge transfer on the sub-3 fs timescale from the LUMO+2 of the biisonicotinic acid molecule into the conduction band of the underlying $\mathrm{TiO}_{2}(110)$ conduction band has been experimentally measured using RPES and is supported by sub-5 fs timescales measured for isonicotinic acid on the same surface. $\frac{6[18}{6}$ However, in our measurements there is no evidence of the channels identified as participator decay processes (elastic scattering and vibronic coupling) being depleated for the monolayer for which fs charge transfer out of the LUMO +2 has been shown to occur. Enhancements of the Rayleigh (elastic) line previously observed in the RIXS of isolated molecules are typically understood in terms of core-excitonic states leading to an enhanced probability of the originally excited electron filling the corehole, resulting in elastic participator decay 16117 Such enhancements as observed here in the multilayer data would be expected to vanish if the originally excited electron tunnels away on a fast timescale in the monolayer.

Since the intermediate state in RIXS and RPES are the same (resonant excitation of the core-electron to the unoccupied valence state) we do not expect differences in the timescales of electron delocalization in the core-excited state between the two techniques. If the radiative core-exciton decay process occurs on a faster timescale it would of course not be depleted by charge transfer on the low femtosecond timescale. However, this is difficult to reconcile 
with the core-hole lifetime of around 6 fs. $\frac{3}{3}$ This suggests instead a challenge for the twostep model of RIXS that is implicit in the core-hole clock approach, instead requiring a full quantum mechanical description of RIXS as a coherent one-step process. It is also possible that the measurement statistics in this experiment are insufficient to detect the depletion of the participator channel in the RIXS. Further experiments are required to explore whether the participator channel behaves differently to RPES in other molecule-surface systems.

One candidate is $\mathrm{C}_{60}$ on $\mathrm{Au}(111)$ for which the RPES data has already been measured.20 This system has the advantage of substantially more probed atoms per molecule, and more intense LUMO+1, 2 and 3 resonances relative to the LUMO. Moreover, the core-excited LUMO straddles the Fermi level of the metal surface opening up the possibility for charge transfer out of those vibrational states that overlap with empty densities of states in the surface and modification of the vibronic coupling ${ }^{\sqrt{15}}$ contribution to the participator channel.

\section{CONCLUSIONS}

Resonant inelastic x-ray scattering has been used to study the excited-state charge transfer dynamics of an aromatic molecule, biisonicotinic acid, coupled to a rutile $\mathrm{TiO}_{2}(110)$ single crystal surface. By measuring, for the first time, full RIXS maps for both multilayer and monolayer coverages of the molecules, the participator channel can be clearly identified and quantified as a function of excitation into different unoccupied molecular orbitals. Included in this quantification is the elastic line and the inelastic scattering due to ultra-fast vibronic coupling on the timescale of the core-hole lifetime. The orbital-specific participator channel has been probed in the absence of charge transfer in the physisobred multilayer, and compared to the coupled molecules in the chemisorbed monolayer. A core-hole clock analysis of the RIXS data in order to extract a charge transfer time for the LUMO+2 excited state that lies above the conduction band edge has been performed. However, there is no compelling evidence in the data to show that participator channel is depleted under the conditions of ultra-fast charge transfer of the excited electron into the substrate. The data instead suggests that the processes that give rise to both the elastic scattering and the intramolecular vibronic coupling, both of which are participator processes are not modified on the femtosecond timescale on which charge transfer out of the LUMO+2 to the conduction band of $\mathrm{TiO}_{2}(110)$ is already established. 


\section{ACKNOWLEDGEMENTS}

Funding was provided by RCUK — Engineering and Physical Sciences Research Council (EPSRC) and Molecularspray Ltd through a DTG/CASE conversion studentship.

\section{REFERENCES}

${ }^{1}$ M. Grätzel, Journal of Photochemistry and Photobiology C: Photochemistry Reviews 4, 145 (2003).

${ }^{2}$ J. J. Concepcion, J. W. Jurss, M. K. Brennaman, P. G. Hoertz, A. O. T. Patrocinio, N. Y. Murakami Iha, J. L. Templeton, and T. J. Meyer, Acc. Chem. Res. 42, 1954 (2009).

${ }^{3}$ B. Kempgens, A. Kivimäki, M. Neeb, H. M. Köppe, A. M. Bradshaw, and J. Feldhaus, Journal of Physical Chemistry B 29, 5389 (1996).

${ }^{4}$ P. A. Brühwiler, O. Karis, and N. Mårtensson, Reviews of Modern Physics 74, 703 (2002).

${ }^{5}$ Participator has become the accepted term in the literature to describe the core-hole decay process in which the originally excited electron is a direct participant, resulting in resonant photoemission in RPES and inelastic scattering in RIXS. In Ref. 4 by P. A. Brühwiler et al, the grammatically correct term participant is used instead. Both terms refer to the same processes.

${ }^{6}$ J. Schnadt, P. A. Brühwiler, L. Patthey, J. N. O’Shea, S. Södergren, M. Odelius, R. Ahuja, O. Karis, M. Bässler, P. Persson, H. Siegbahn, S. Lunell, and N. Mårtensson, Nature 418, $620(2002)$.

${ }^{7}$ L. Patthey, H. Rensmo, P. Persson, K. Westermark, L. Vayssieres, A. Stashans, A. Petersson, P. A. Brühwiler, H. Siegbahn, S. Lunell, and N. Märtensson, J. Chem. Phys. 110, 5913 (1999).

${ }^{8}$ L. C. Mayor, J. B. Taylor, G. Magnano, A. Rienzo, C. J. Satterley, J. N. O’Shea, and J. Schnadt, J. Chem. Phys. 129, 114701 (2008).

${ }^{9}$ M. Weston, A. J. Britton, and J. N. O’Shea, J. Chem. Phys 134, 054705 (2011).

${ }^{10}$ M. Weston, K. Handrup, T. J. Reade, N. R. Champness, and J. N. O'Shea, J. Chem. Phys. 137, 224706 (2012).

${ }^{11}$ A. J. Britton, M. Weston, and J. N. O'Shea, Phys. Rev. Letters 109, 017401 (2012). 
${ }^{12}$ S. G. Chiuzbaian, C. F. Hague, A. Avila, R. Delaunay, N. Jaouen, M. Sacchi, F. Polack, M. Thomasset, B. Lagarde, A. Nicolaou, S. Brignolo, C. Baumier, J. Luening, and J.-M. Mariot, Rev. Sci. Instrum. 85, 043108 (2014).

${ }^{13}$ M. Sacchi, N. Jaouen, H. Popescu, R. Gaudemer, J. M. Tonnerre, S. G. Chiuzbaian, C. F. Hague, A. Delmotte, J. M. Dubuisson, G. Cauchon, B. Lagarde, and F. Polack, in 11th International Conference on Synchrotron Radiation Instrumentation (SRI 2012), J. Phys. Conf. Ser., Vol. 425, edited by Susini, J and Dumas, P (2013) p. 072018.

${ }^{14}$ P. Persson, S. Lunell, P. Bruhwiler, J. Schnadt, S. Sodergren, J. O’Shea, O. Karis, H. Siegbahn, N. Martensson, M. Bassler, and L. Patthey, J. Chem. Phys. 112, 3945 (2000).

${ }^{15}$ L. Kjeldgaard, T. Kaambre, J. Schiessling, I. Marenne, J. O’Shea, J. Schnadt, C. Glover, M. Nagasono, D. Nordlund, M. Garnier, L. Qian, J. Rubensson, P. Rudolf, N. Martensson, J. Nordgren, and P. Bruhwiler, Phys. Rev. B 72, 205414 (2005).

${ }^{16}$ L. Weinhardt, O. Fuchs, D. Batchelor, M. Baer, M. Blum, J. D. Denlinger, W. Yang, A. Schoell, F. Reinert, E. Umbach, and C. Heske, J. Chem. Phys. 135, 104705 (2011).

${ }^{17}$ L. Weinhardt, E. Ertan, M. Iannuzzi, M. Weigand, O. Fuchs, M. Bar, M. Blum, J. D. Denlinger, W. Yang, E. Umbach, M. Odelius, and C. Heske, Phys. Chem. Chem. Phys. 17, 27145 (2015).

${ }^{18}$ J. Schnadt, J. N. O’Shea, L. Patthey, L. Kjeldgaard, J. Åhlund, K. Nilson, J. Schiessling, J. Krempaský, M. Shi, O. Karis, C. Glover, H. Siegbahn, N. Mårtensson, and P. A. Brühwiler, J. Chem. Phys. 119, 12462 (2003).

${ }^{19}$ J. Schnadt, J. O’Shea, L. Patthey, J. Krempasky, N. Martensson, and P. Bruhwiler, Phys. Rev. B 67, 235420 (2003).

${ }^{20}$ A. J. Gibson, R. H. Temperton, K. Handrup, and J. N. O’Shea, Surf. Sci. 657, 69 (2017). 